\title{
Evaluation of risk factors and causative pathogens in bloodstream infections in cancer patients
}

\author{
(1) Duygu Mert', (1) Alparslan Merdin², (1) Nurten Kandemir ${ }^{3}$, (1) Merih Kızıl Çakar², (1) Ece Dirim \\ (1) Sabahat Çeken ${ }^{1}$, (1) Fevzi Altuntaş ${ }^{2}$, (1) Mustafa Ertek ${ }^{1}$
}

1 University of Health Sciences Turkey, Dr. Abdurrahman Yurtaslan Ankara Oncology Training and Research Hospital, Clinic of Infectious Diseases and Clinical Microbiology, Ankara, Turkey

2University of Health Sciences Turkey, Dr. Abdurrahman Yurtaslan Ankara Oncology Training and Research Hospital, Clinic of Hematology and Bone Marrow Transplantation Unit, Ankara, Turkey

3University of Health Sciences Turkey, Dr. Abdurrahman Yurtaslan Ankara Oncology Training and Research Hospital, Clinic of Medical Oncology, Ankara, Turkey

4 University of Health Sciences Turkey, Dr. Abdurrahman Yurtaslan Ankara Oncology Training and Research Hospital, Microbiology Laboratory, Ankara, Turkey

\section{Date submitted:}

19.08.2020

Date accepted:

22.10.2020

Online publication date:

15.03.2021

\section{Corresponding Author:}

Duygu Mert MD, University of Health Sciences Turkey, Dr. Abdurrahman Yurtaslan Ankara Oncology Training and Research Hospital, Clinic of Infectious Diseases and Clinical Microbiology, Ankara, Turkey

drduygumert@hotmail.com

ORCID:

orcid.org/0000-0002-6810-2199

Keywords: Bloodstream infection, hematologic malignancy, solid tumor

\begin{abstract}
Aims: Bloodstream infection in cancer patients may cause delays in chemotherapy, prolonged hospital stay, difficulties in diagnosis and treatment, inappropriate treatment, high mortality rates and increased health care costs. The aim of this study was to evaluate the risk factors and causative pathogens in bloodstream infection in cancer patients.
\end{abstract}

Methods: The study was planned as a retrospective case-control study. The study included patients who were followed up and treated with the diagnosis of hematologic malignancy or solid tumor and who were found to have bloodstream infection during the period from $01 / 01 / 2014$ to $12 / 31 / 2018$ and patients with similar characteristics as the control group.

Results: A total of 312 (276 hematologic malignancy, 36 solid tumor) bloodstream infections were included. The presence of central venous catheter (CVC), steroid use, presence of urinary catheter, neutrophil count, neutropenia duration and total hospitalization day were determined as independent risk factors of bloodstream infection in patients with hematologic malignancy. The presence of CVC, total parenteral nutrition, renal failure and neutrophil count were determined as independent risk factors of bloodstream infection in patients with solid tumor. In patients with hematologic malignancy, 113 Escherichia coli and 59 Klebsiella pneumoniae were detected. In patients with solid tumor, 7 E. coli and 5 K. pneumoniae were detected.

Conclusions: Risk factors are higher in the development of bloodstream infection in patients with hematologic malignancy and patients should be closely monitored. Multidrug-resistant bacteria might also be isolated from the blood cultures of patients. Therefore, it is recommended to start with broad-spectrum antibiotics in bloodstream infections.

\section{Introduction}

Despite improvements in antimicrobial treatment and supportive care services, bloodstream infections are an important cause of morbidity and mortality (1). Bloodstream infections in malignant patients are among the life-threatening complications leading to delays in chemotherapy, prolonged hospital stay, difficulties in diagnosis and treatment, inappropriate treatment, high mortality and increased health care costs (2).

The frequency of bloodstream infections is between $11 \%$ and $38 \%$ and the mortality rate reaches $40 \%$ (3). The presence of multidrug-resistant bacteria increases the morbidity and mortality rates in these patients (1). In recent years, Gram- 
negative bacteria have been detected as causative agents, but this varies according to the geographical region (2).

Candidemia means the presence of Candida species in the blood. When Candida is detected in blood cultures, the source of the infection should be investigated. Candidemia is an indicator of disseminated candidiasis in many patients (4).

The aim of this study was to determine the risk factors, the distribution and frequency of isolated agents, and to determine the resistance status in bloodstream infections in cancer patients.

\section{Methods}

The study was planned as a retrospective case-control study. Approval was obtained from the University of Health Sciences Turkey Dr. Abdurrahman Yurtaslan Ankara Oncology Training and Research Hospital Ethics Committee (approval date: 02/20/2019 and decision no: 2019-03/209).

The study included patients who were followed up and treated with the diagnosis of hematologic malignancies and solid tumors and who were found to have bloodstream infections during the period from $01 / 01 / 2014$ to $12 / 31 / 2018$, and patients with similar characteristics but no growth in culture materials were included in the control group.

The control group was selected from the patients who presented to the hospital in the same period, had no clinical signs and symptoms suggestive of bloodstream infection and had negative blood culture. The patients in the study and control groups were matched in terms of hospitalization period and inpatient unit type. The files of the patients were analyzed retrospectively and the data obtained were transferred to the forms for evaluation.

The analysis of the risk factors for the development of bloodstream infections was performed from the data obtained until the development of bloodstream infection. For the control group, risk analysis was planned from the data of hospitalization periods.

When fever was $\geq 38.3^{\circ} \mathrm{C}$ in patients with malignancies, at least 2 vials of blood cultures were drawn from each patient. Blood cultures were obtained from different peripheral veins of patients with central venous catheter (CVC), including one from the catheter.

The automated BacT/ALERT 3D (bioMerieux, Marcyl'Etoile, France) system, which detects the growth of blood cultures by signal, was used. Antibiotic susceptibility tests of factors were performed according to the recommendations of the European Committee on Antimicrobial Susceptibility Testing (EUCAST). According to the Center for Disease Control and Prevention (CDC) definitions, the patients were divided into two groups as primary bloodstream infections and catheter-related bloodstream infections.
The blood samples were inoculated into automated BacT/ ALERT 3D (bioMerieux, Marcy-l'Etoile, France) system for blood cultures. C. albicans were separated by using the germ tube test for typing. The non-albicans species were typed with VITEK ${ }^{\circledR}$ 2 Compact System (bioMerieux, France). Amphotericin B, fluconazole, flucytosine, voriconazole, caspofungin, itraconazole sensitivity tests were performed with VITEK ${ }^{\circledR} 2$ Compact System (bioMerieux, France). EUCAST was used in the antifungal test.

Results of the patients were recorded electronically from the health records. The demographic data of the patients and their other information at the time of cultures were recorded in a preprepared form. Patients with microbial growth in their blood and/ or catheter cultures were included in the study.

Eighteen-year-old and older patients with malignancies, who had clinical, laboratory and microbiological bloodstream infection history, were included in the study. Patients who were under the age of 18 years, pregnant patients and those without malignancies were excluded from the study.

\section{Statistical Analysis}

SPSS (IBM SPSS Statistics 24) program was used in the statistical analyses. The data were entered into statistical software program and analyzed by using the same computer software program. In order to interpret the findings, frequency tables and descriptive statistics were used.

The categorical descriptive data were presented as frequency distribution and percentages (\%) and the measurable descriptive data were presented as mean \pm standard deviation and median (the largest, the smallest values).

The " $x^{2}$-cross tables" were used to evaluate the relationship between two qualitative variables. The "Mann-Whitney U" test (Z-table value) statistics were used to compare the two nonnormally distributed independent groups. The "Binary Logistic Regression" analysis was used to determine independent risk factors. $P<0.05$ was considered statistically significant.

\section{Results}

The data of 276 patients with hematologic malignancy and bloodstream infections and data of 36 patients with solid tumor and bloodstream infections were evaluated.

A total of 312 (276 hematologic malignancy, 36 solid tumor) bloodstream infections were detected in these patients. Ninety seven $(44.3 \%)$ of the patients with hematologic malignancy were female and $122(55.7 \%)$ were male. The mean age of the patients was $44.5 \pm 16.2$ years. One hundred-nine $(49.7 \%)$ of the study (case) group patients had acute myeloid leukemia, $48(21.8 \%)$ had non-Hodgkin lymphoma and $44(20.1 \%)$ had acute lymphoblastic leukemia (Table 1). Sixteen $(44.4 \%)$ of the patients with solid tumor were female and 20 (55.6\%) were male. The mean age of the patients was $56.0 \pm 14.4$ years. Thirty six $(100 \%)$ patients had solid tumors (Table 2 ). 


\begin{tabular}{|c|c|c|c|}
\hline & $\begin{array}{l}\text { Patients with hematologic } \\
\text { malignancy }(n=276, \%)\end{array}$ & Control $(n=153, \%)$ & $\mathrm{p}$ value \\
\hline \multicolumn{4}{|l|}{ Gender } \\
\hline Female & $97(44.3 \%)$ & $55(35.9 \%)$ & \multirow{2}{*}{$\begin{array}{l}x^{2}=2.595 \\
p=0.107\end{array}$} \\
\hline Male & $122(55.7 \%)$ & $98(64.1 \%)$ & \\
\hline \multicolumn{4}{|l|}{ Disease } \\
\hline AML & $109(49.7 \%)$ & $70(45.8 \%)$ & \multirow{9}{*}{$\begin{array}{l}x^{2}=7.538 \\
p=0.480\end{array}$} \\
\hline ALL & $44(20.1 \%)$ & $29(19.0 \%)$ & \\
\hline CML & $3(1.4 \%)$ & - & \\
\hline CLL & $1(0.5 \%)$ & - & \\
\hline $\mathrm{HCL}$ & $1(0.5 \%)$ & - & \\
\hline MM & $8(3.7 \%)$ & $4(2.6 \%)$ & \\
\hline MDS & $4(1.8 \%)$ & $4(2.6 \%)$ & \\
\hline $\mathrm{NHL}$ & $48(21.8 \%)$ & $45(29.4 \%)$ & \\
\hline $\mathrm{HL}$ & $1(0.5 \%)$ & $1(0.6 \%)$ & \\
\hline Hospitalization in the last three months & $155(56.2 \%)$ & $85(58.6 \%)$ & $\begin{array}{l}X^{2}=1.840 \\
p=0.399\end{array}$ \\
\hline Intensive care unit stay & $20(7.2 \%)$ & $4(2.6 \%)$ & $\begin{array}{l}x^{2}=3.170 \\
p=0.075\end{array}$ \\
\hline Presence of central venous catheter & $167(60.5 \%)$ & $42(27.5 \%)$ & $\begin{array}{l}x^{2}=43.052 \\
p<0.001\end{array}$ \\
\hline Total parenteral nutrition & $37(13.5 \%)$ & $3(2.0 \%)$ & $\begin{array}{l}x^{2}=14.002 \\
p<0.001\end{array}$ \\
\hline Renal failure & $29(10.5 \%)$ & $2(1.3 \%)$ & $\begin{array}{l}X^{2}=11.093 \\
p<0.001\end{array}$ \\
\hline Diabetes mellitus & $29(10.5 \%)$ & $10(6.5 \%)$ & $\begin{array}{l}X^{2}=1.429 \\
p=0.232\end{array}$ \\
\hline HSCT (allogeneic, autologous) & $22(8.0 \%)$ & $14(9.2 \%)$ & $\begin{array}{l}X^{2}=0.058 \\
p=0.810\end{array}$ \\
\hline Allogeneic HSCT & $15(5.4 \%)$ & $11(7.2 \%)$ & $\begin{array}{l}X^{2}=0.269 \\
p=0.604\end{array}$ \\
\hline Autologous HSCT & $10(3.6 \%)$ & $3(2.0 \%)$ & $p=0.258$ \\
\hline \multicolumn{4}{|l|}{ Disease status } \\
\hline Newly diagnosed & $98(35.5 \%)$ & $50(32.7 \%)$ & \multirow{6}{*}{$\begin{array}{l}x^{2}=7.817 \\
p=0.167\end{array}$} \\
\hline Remission & $74(26.8 \%)$ & $58(37.9 \%)$ & \\
\hline Refractory & $30(10.9 \%)$ & $15(9.8 \%)$ & \\
\hline Relapse & $58(21.0 \%)$ & $265(13.1 \%)$ & \\
\hline Stable & $14(5.1 \%)$ & $20(5.9 \%)$ & \\
\hline Not evaluated group & $2(0.7 \%)$ & $9(0.6 \%)$ & \\
\hline \multicolumn{4}{|l|}{ Name of the given chemotherapy regimen } \\
\hline Induction & $96(35.8 \%)$ & $47(37.0 \%)$ & \multirow{4}{*}{$\begin{array}{l}x^{2}=7.574 \\
p=0.056\end{array}$} \\
\hline Consolidation & $83(31.0 \%)$ & $53(41.7 \%)$ & \\
\hline Salvage regimen & $83(31.0 \%)$ & $24(18.9 \%)$ & \\
\hline Palliative chemotherapy or other chemotherapies & $6(2.2 \%)$ & $3(2.4 \%)$ & \\
\hline Steroid use & $77(27.9 \%)$ & $20(13.1 \%)$ & $\begin{array}{l}X^{2}=15.141 \\
p=0.001\end{array}$ \\
\hline Quinolone prophylaxis & $31(11.2 \%)$ & $14(9.2 \%)$ & $\begin{array}{l}x^{2}=0.260 \\
p=0.610\end{array}$ \\
\hline
\end{tabular}




\begin{tabular}{|c|c|c|c|}
\hline Antibiotic use in the last three months & $139(50.4 \%)$ & $49(32.0 \%)$ & $\begin{array}{l}x^{2}=3.443 \\
p<0.001\end{array}$ \\
\hline Another bloodstream infection in the last three months & $71(25.7 \%)$ & $8(5.2 \%)$ & $\begin{array}{l}x^{2}=27.523 \\
p<0.001\end{array}$ \\
\hline Presence of another infection & $85(30.8 \%)$ & $28(18.3 \%)$ & $\begin{array}{l}X^{2}=7.923 \\
p=0.005\end{array}$ \\
\hline Presence of urinary catheter & $48(17.4 \%)$ & $5(3.3 \%)$ & $\begin{array}{l}X^{2}=16.852 \\
p<0.001\end{array}$ \\
\hline Presence of mucositis & $201(72.8 \%)$ & $84(54.9 \%)$ & $\begin{array}{l}X^{2}=14.182 \\
p<0.001\end{array}$ \\
\hline Additional disease & $76(27.5 \%)$ & $41(26.8 \%)$ & $\begin{array}{l}x^{2}=0.027 \\
p=0.869\end{array}$ \\
\hline \multicolumn{4}{|l|}{ Discharge status } \\
\hline Discharge & $211(76.4 \%)$ & $147(96.1 \%)$ & \multirow{2}{*}{$\begin{array}{l}x^{2}=27.461 \\
p<0.001\end{array}$} \\
\hline Mortality & $65(23.6 \%)$ & $6(3.9 \%)$ & \\
\hline \multicolumn{4}{|c|}{$\begin{array}{l}\text { The " }{ }^{2} \text {-cross tables" were used to investigate the relationship between two qualitative variables. } \\
\text { AML: Acute myeloid leukemia, ALL: Acute lymphoblastic leukemia, CML: Chronic myeloid leukemia, CLL: Chronic lymphocytic leukaemia, HCL: Hairy cell leukemia, } \\
\text { MM: Multiple myeloma, MDS: Myelodysplastic syndrome, NHL: Non-Hodgkin lymphoma, HL: Hodgkin lymphoma, HSCT: Hematopoietic stem cell transplantation }\end{array}$} \\
\hline
\end{tabular}

The hospitalization status in the last three months, intensive care unit stay, hematopoietic stem cell transplantation, disease status, given chemotherapy regimens, quinolone use and additional disease were not significantly different between the patients with hematologic malignancy and the control group in patients with hematological malignancy (Table 1). The hospitalization status in the last three months, given chemotherapy regimens, steroid use, antibiotic use in the last three months and additional disease were not significantly different between the patients with solid tumor and the control group in patients with solid tumor (Table 2).

There was a significant difference in the presence of CVC between the patients with hematologic malignancy and the control group in patients with hematologic malignancy $(p<0.001)$. The patients [167 (60.5\%)] had CVC, $51(18.5 \%)$ were ports and $116(42.8 \%)$ were permanent central catheters. A significant difference wasalso found between the patients with solid tumor and the control group in terms of the presence of CVC in patients with solid tumor $(p<0.001)$. Twenty-seven $(75 \%)$ patients had CVC; 16 (44.4\%) were port and 11 (30.6\%) were permanent central catheter.

The total parenteral nutrition (TPN) history, renal failure, steroid use, antibiotic use in the last three months, another bloodstream infection in the last three months, presence of another infection, presence of urinary catheter, mucositis and mortality statuses were significantly different between the patients with hematologic malignancy and the control group in bloodstream infections $(p<0.001, p=0.001, p=0.001, p<0.001$, $p<0.001, p=0.005, p<0.001, p<0.001$ and $p<0.001$, respectively) (Table 1). The intensive care unit stay, TPN history, renal failure, disease status, another bloodstream infection in the last three months, presence of another infection, presence of urinary catheter, mucositis and mortality statuses were significantly different between the patients with solid tumor and the control group in bloodstream infections $(p=0.005, p=0.001, p<0.001$, $p=0.013, p=0.025, p<0.001, p<0.001, p=0.006$ and $p=0.005$, respectively) (Table 2 ).

There was no significant difference in terms of diabetes mellitus between the patients with hematologic malignancy and the control group ( $p=0.232)$. There was no significant difference in terms of diabetes mellitus between the patients with solid tumor and the control group $(p=0.343)$.

The mean neutrophil count was $30 / \mathrm{mm}^{3}$ in the patients with hematologic malignancy and the mean neutrophil count was $600 / \mathrm{mm}^{3}$ in the control group. There was a statistically significant difference $(p<0.001)$. There was a statistically significant difference in terms of neutropenia duration and total hospitalization days between the patients with hematologic malignancy and the control group $(p<0.001$ and $p<0.001$, respectively) (Table 3 ). The mean neutrophil count was 4.395/ $\mathrm{mm}^{3}$ in the patients with solid tumor and $3.460 / \mathrm{mm}^{3}$ in the control group ( $p=0.470)$. There was a statistically significant difference in terms of neutropenia duration and total hospitalization day between the patients with solid tumor and the control group $(p=0.010$ and $p<0.001$, respectively) (Table 3 ).

The mean duration of neutropenia before infection was $9.5 \pm 9.6$ (7.0) days and the mean of total hospitalization days before infection was 18.4 days in the patients with hematologic malignancy (Table 3). The mean duration of neutropenia before infection was $1.2 \pm 2.0(1.0)$ days and the mean of total hospitalization days before infection was 8.7 days in the patients with solid tumor (Table 3).

The presence of CVC, steroid use, antibiotic use in last three months, presence of urinary catheter, neutrophil count, neutropenia duration and total hospitalization days 


\begin{tabular}{|c|c|c|c|}
\hline & Patients with solid tumor $(n=36, \%)$ & Control $(n=37, \%)$ & $\mathrm{p}$ value \\
\hline \multicolumn{4}{|l|}{ Gender } \\
\hline Female & $16(44.4 \%)$ & $16(43.2 \%)$ & \multirow{2}{*}{$\begin{array}{l}x^{2}=0.000 \\
p=1.000\end{array}$} \\
\hline Male & $20(55.6 \%)$ & $21(56.8 \%)$ & \\
\hline \multicolumn{4}{|l|}{ Disease } \\
\hline Solid tumor & $36(100 \%)$ & $37(100 \%)$ & $\begin{array}{l}x^{2}=0.004 \\
p=0.998\end{array}$ \\
\hline Hospitalization in the last three months & $17(47.2 \%)$ & $21(556.8 \%)$ & $\begin{array}{l}x^{2}=0.337 \\
p=0.561\end{array}$ \\
\hline Intensive care unit stay & $7(19.4 \%)$ & - & $p=0.005$ \\
\hline Presence of central venous catheter & $27(75.0 \%)$ & $1(29.7 \%)$ & $\begin{array}{l}x^{2}=13.224 \\
p<0.001\end{array}$ \\
\hline Total parenteral nutrition & $15(41.7 \%)$ & $2(5.4 \%)$ & $\begin{array}{l}X^{2}=11.477 \\
p=0.001\end{array}$ \\
\hline Renal failure & $17(47.2 \%)$ & $1(2.7 \%)$ & $\begin{array}{l}X^{2}=17.144 \\
p<0.001\end{array}$ \\
\hline Diabetes mellitus & $9(25.0 \%)$ & $5(13.5 \%)$ & $\begin{array}{l}x^{2}=0.901 \\
p=0.343\end{array}$ \\
\hline \multicolumn{4}{|l|}{ Disease status } \\
\hline Remission & $1(2.8 \%)$ & - & \multirow{4}{*}{$\begin{array}{l}x^{2}=10.766 \\
p=0.013\end{array}$} \\
\hline Refractory & - & $1(2.7 \%)$ & \\
\hline Stable & - & $8(21.6 \%)$ & \\
\hline Metastatic & $35(97.2 \%)$ & $28(75.7 \%)$ & \\
\hline \multicolumn{4}{|l|}{ Name of the given chemotherapy regimen } \\
\hline Consolidation & - & $1(4.2 \%)$ & \multirow{4}{*}{$\begin{array}{l}x^{2}=6.396 \\
p=0.094\end{array}$} \\
\hline Salvage regimen & - & $1(4.2 \%)$ & \\
\hline Palliative chemotherapy & $26(96.3 \%)$ & $17(70.8 \%)$ & \\
\hline Other chemotherapies & $1(3.7 \%)$ & $5(20.8 \%)$ & \\
\hline Steroid use & $5(13.9 \%)$ & $1(2.7 \%)$ & $p=0.093$ \\
\hline Antibiotic use in last three months & $12(33.3 \%)$ & $5(13.5 \%)$ & $\begin{array}{l}x^{2}=2.980 \\
p=0.084\end{array}$ \\
\hline Another bloodstream infection in the last three months & $5(13.9 \%)$ & - & $p=0.025$ \\
\hline Presence of another infection & $16(44.4 \%)$ & $2(5.4 \%)$ & $\begin{array}{l}X^{2}=12.941 \\
p<0.001\end{array}$ \\
\hline Presence of urinary catheter & $16(44.4 \%)$ & $2(5.4 \%)$ & $\begin{array}{l}x^{2}=12.941 \\
p<0.001\end{array}$ \\
\hline Presence of mucositis & $1(33.3 \%)$ & $2(5.4 \%)$ & $\begin{array}{l}X^{2}=7.46 \\
p=0.006\end{array}$ \\
\hline Additional disease & $15(41.7 \%)$ & $12(32.4 \%)$ & $\begin{array}{l}x^{2}=0.330 \\
p=0.566\end{array}$ \\
\hline $\begin{array}{l}\text { Discharge status } \\
\text { Discharge }\end{array}$ & $29(80.6 \%)$ & $37(100 \%)$ & \multirow[t]{2}{*}{$p=0.005$} \\
\hline Mortality & $7(19.4 \%)$ & - & \\
\hline
\end{tabular}

were determined as independent risk factors of bloodstream infection in the patients with hematologic malignancy (Table 4). The presence of CVC, history of TPN renal failure and neutrophil count were determined as independent risk factors of bloodstream infection in the patients with solid organ tumor (Table 4).
In the patients with hematologic malignancy, $113(51.7 \%)$ Escherichia coli, 59 (27.1\%) Klebsiella pneumoniae and 19 (8.6\%) Pseudomonas aeruginosa were found to be positive for Gram-negative bacteria. Extended-spectrum beta-lactamase (ESBL) positivity was found to be $110(50.5 \%)$, carbapenemase 


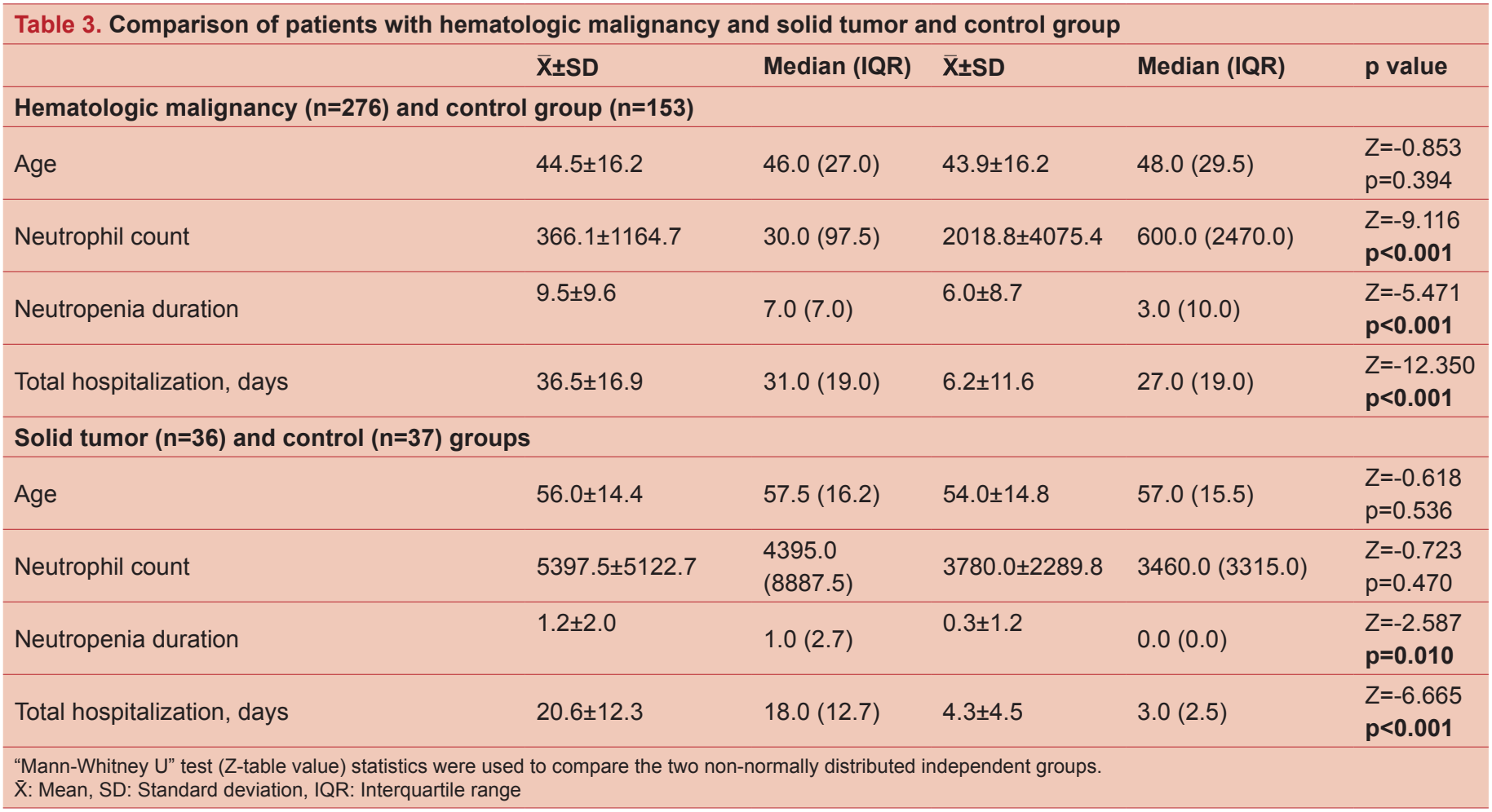

\begin{tabular}{|c|c|c|c|c|c|c|c|c|}
\hline & \multirow{2}{*}{ B } & \multirow{2}{*}{$\begin{array}{l}\text { Standard } \\
\text { error }\end{array}$} & \multirow{2}{*}{ Wald } & \multirow{2}{*}{ df } & \multirow{2}{*}{ Sig. } & \multirow{2}{*}{ OR } & \multicolumn{2}{|l|}{$95 \% \mathrm{Cl}$} \\
\hline & & & & & & & Lower & Upper \\
\hline Presence of central venous catheter & 1.0 & 0.3 & 9.5 & 1 & 0.002 & 2.7 & 1.4 & 5.0 \\
\hline Steroid use & 1.9 & 0.5 & 13.5 & 1 & $<0.001$ & 6.6 & 2.4 & 18.2 \\
\hline Neutrophil count & -0.001 & 0.000 & 14.1 & 1 & $<0.001$ & 0.9 & 0.9 & 0.9 \\
\hline Neutropenia duration & -0.140 & 0.0 & 26.6 & 1 & $<0.001$ & 0.9 & 0.8 & 0.9 \\
\hline Total hospitalization, days & 0.2 & 0.0 & 61.6 & 1 & $<0.001$ & 1.2 & 1.1 & 1.2 \\
\hline \multicolumn{9}{|l|}{$\mathrm{CCR}=87.4 \% ; \chi_{(8)}^{2}=5.923, p=0.656$} \\
\hline Renal failure & 4.3 & 1.4 & 9.7 & 1 & 0.002 & 76.9 & 5.0 & 174.2 \\
\hline Presence of urinary catheter & 2.7 & 1.5 & 3.4 & 1 & 0.063 & 15.6 & 0.8 & 283.8 \\
\hline Neutrophil count & 0.05 & 0.02 & 7.1 & 1 & 0.008 & 1.05 & 1.1 & 1.9 \\
\hline \multicolumn{9}{|l|}{$\mathrm{CCR}=86.3 \% ; \chi_{(8)}^{2}=4.032, p=0.854$} \\
\hline
\end{tabular}

positivity was found to be $39(17.9 \%)$ and multidrug resistance was found to be $122(56.0 \%)$. In the patients with solid tumor, $7(41.2 \%)$ E. coli, 5 (29.4\%) K. pneumoniae and 3 (17.6\%) P. aeruginosa were found to be positive for Gram-negative bacteria. ESBL positivity was found to be $8(47.1 \%)$, carbapenemase positivity was found to be $1(5.9 \%)$ and multidrug resistance was found to be $5(29.4 \%)$ (Table 5$)$.

In the patients with hematologic malignancy, 20 (38.6\%) Staphylococcus epidermidis was the most common causative agent in Gram-positive bacteria, followed by $13(25.0 \%)$ 
Staphylococcus hominis and 6 (11.5\%) Staphylococcus haemolyticus. Methicillin resistance 44 (84.6\%), penicillin resistance $46(88.5 \%)$ and vancomycin resistance 1 (1.9\%) were found. In the patients with solid tumor, $4(25.0 \%) \mathrm{S}$. epidermidis were the most common causative agent in Grampositive bacteria, followed by 3 (18.7\%) Staphylococcus aureus and $3(18.7 \%)$ Enterococcus faecium. Methicillin resistance $10(62.5 \%)$, penicillin resistance $13(81.2 \%)$ and vancomycin resistance 1 (6.3\%) were found (Table 5).

In the patients with hematologic malignancy, two Candida krusei and Candida tropicalis were isolated, whereas Candida albicans was isolated in only one patient. All of the fungal agents were susceptible to voriconazole, one was moderately susceptible to amphotericin, and two were moderately susceptible to flucytosine. All of the fungal agents were not resistant to caspofungin and micafungin and two of them were resistant to fluconazole. One C. albicans and one C. tropicalis were isolated in patients with solid tumor. It was only moderately sensitive to $C$. tropicalis amphotericin and there was no resistance to all other antifungal drugs in both fungal agents.

\section{Discussion}

In patients with malignancy, the immunosuppressive effects of chemotherapy as well as immune defects due to underlying hematological disorders increase the risk of infection in patients (5). In addition, the application of chemotherapy does not only reduce the number of neutrophils, but also cause chemotactic and phagocytic disorders (6).

The incidence of bloodstream infections in patients with malignancy is $11.7 \%$ and this rate is reported to be between $11.8 \%$ and $33.3 \%$ in different geographical regions (7).

In one study, the incidence of bloodstream infection was found to be eight times higher in the patients with hematologic malignancy than in the patients with solid tumor due to the underlying disease (2). In this study, 276 bloodstream infection were detected in the patients with hematologic malignancy and 36 bloodstream infection in the patients with solid tumor.

The secondary bloodstream infection and catheter-related bloodstream infection are more common in the patients with solid tumor, whereas primary and mucosity-related bloodstream infections are more common in the patients with hematologic malignancy (2). In this study, primary bloodstream infection was found to be $161(58.3 \%)$, catheter-related bloodstream infection was found to be $115(41.7 \%)$ in the patients with hematologic malignancy, whereas primary bloodstream infection was found to be $18(50 \%)$, catheter-related bloodstream infection was found to be $18(50 \%)$ in the patients with solid tumor. Primary bloodstream infection rate was found to be higher in patients with hematologic malignancy.

\begin{tabular}{|c|c|c|}
\hline $\begin{array}{l}\text { Variable (patients with hematologic } \\
\text { malignancy) }\end{array}$ & $\mathbf{n}$ & $\%$ \\
\hline \multicolumn{3}{|l|}{ Gram-negative bacteria } \\
\hline Escherichia coli & 113 & 51.7 \\
\hline Klebsiella pneumoniae & 59 & 27.1 \\
\hline Pseudomonas aeruginosa & 19 & 8.6 \\
\hline Enterobacter cloacae & 6 & 2.8 \\
\hline Acinetobacter baumannii & 7 & 3.2 \\
\hline Aeromonas hydrophila & 3 & 1.4 \\
\hline Pseudomonas spp. & 5 & 2.3 \\
\hline Sphingomonas paucimobilis & 1 & 0.5 \\
\hline Klebsiella oxytoca & 1 & 0.5 \\
\hline Stenotrophomonas maltophilia & 3 & 1.4 \\
\hline Acinetobacter Iwoffii & 1 & 0.5 \\
\hline Extended-spectrum beta-lactamase & 110 & 50.5 \\
\hline Carbapenemase & 39 & 17.9 \\
\hline Multi-drug resistance & 122 & 56.0 \\
\hline \multicolumn{3}{|l|}{ Variable (patients with solid tumor) } \\
\hline \multicolumn{3}{|l|}{ Gram-negative bacteria } \\
\hline Escherichia coli & 7 & 41.2 \\
\hline Klebsiella pneumoniae & 5 & 29.4 \\
\hline Pseudomonas aeruginosa & 3 & 17.6 \\
\hline Klebsiella oxytoca & 1 & 5.9 \\
\hline Stenotrophomonas maltophilia & 1 & 5.9 \\
\hline Extended-spectrum beta-lactamase & 8 & 47.1 \\
\hline Carbapenemase & 1 & 5.9 \\
\hline Multi-drug resistance & 5 & 29.4 \\
\hline \multicolumn{3}{|c|}{ Variable (patients with hematologic malignancy) } \\
\hline \multicolumn{3}{|l|}{ Gram-positive bacteria } \\
\hline Staphylococcus haemolyticus & 6 & 11.5 \\
\hline Corynebacterium spp. & 2 & 3.8 \\
\hline Staphylococcus epidermidis & 20 & 38.6 \\
\hline Enterococcus faecium & 3 & 5.8 \\
\hline Staphylococcus mitis & 2 & 3.8 \\
\hline Staphylococcus hominis & 13 & 25.0 \\
\hline Staphylococcus aureus & 3 & 5.8 \\
\hline Staphylococcus warneri & 1 & 1.9 \\
\hline Kocuria kristinae & 1 & 1.9 \\
\hline Streptococcus spp. & 1 & 1.9 \\
\hline Methicillin resistance & 44 & 84.6 \\
\hline Penicillin resistance & 46 & 88.5 \\
\hline Vancomycin resistance & 1 & 1.9 \\
\hline \multicolumn{3}{|l|}{ Variable (patients with solid tumor) } \\
\hline \multicolumn{3}{|l|}{ Gram-positive bacteria } \\
\hline Staphylococcus epidermidis & 4 & 25.0 \\
\hline
\end{tabular}




\begin{tabular}{|lll|}
\hline Enterococcus faecium & 3 & 18.7 \\
\hline Staphylococcus hominis & 2 & 12.5 \\
\hline Staphylococcus aureus & 3 & 18.7 \\
\hline Enterococcus faecalis & 1 & 6.3 \\
\hline Streptococcus spp. & 2 & 12.5 \\
\hline Leuconostoc spp. & 1 & 6.3 \\
\hline Methicillin resistance & 10 & 62.5 \\
\hline Penicillin resistance & 13 & 81.2 \\
\hline Vancomycin resistance & 1 & 6.3 \\
\hline
\end{tabular}

The risk factors for bloodstream infection caused by Gram-negative bacteria include bone marrow transplantation, liver failure, lower respiratory tract diseases, renal failure, immunsupresif treatments, diabetes mellitus and invazive procedures of uriner tract (8-11). The risk factors for bloodstream infection might include weight loss, inability to feed, wounds on the mucosa and skin, operations of urinary tract and gastrointestinal tract, obstructions, immunosupresif treatments in patients with solid tumor (12). Operations, old age, additional diseases, advanced stage of cancer could be risk factors for bloodstream infection in some hospitals (13).

In patients with hematologic malignancy and patients with solid tumor, the presence of CVC, TPN renal failure and another bloodstream infection in the last three months were found to be risk factors for bloodstream infection in this study $(p=0.013)$. The presence of CVC, steroid use, antibiotic use in last three months, presence of urinary catheter, neutrophil count, neutropenia duration and total hospitalization day were determined as independent risk factors of bloodstream infection in the patients with hematologic malignancy. The presence of CVC, TPN, renal failure and neutrophil count were determined as independent risk factors of bloodstream infection in the patients with solid organ tumor.

Although Gram-negative bacilli were common causative agents in the patients with malignancy in previous studies in the 1960's and 1970's, recent studies have reported that Gram-positive bacteria might be the most common causative agents $(14,15)$. In this study, Gram-negative bacilli were found to be the most common factors in the patients with hematologic malignancy and/or solid tumor.

$E$. coli is the most frequently isolated bacteria. In contrast, $P$. aeruginosa were reported to be the most commonly isolated agent from bloodstream infection in the patients with malignancy in different countries $(16,17)$. In this study, $E$. coli was the most frequently isolated bacteria in both patient groups, followed by $K$. pneumoniae and $P$. aeruginosa.

In a study, the rate of ESBL producing Enterobacteriaceae strains was reported to be $34.1 \%$ in the patients with hematologic malignancy and $65.9 \%$ in the patients with solid tumor (16). In this study, ESBL positivity was found to be $50.5 \%$ in the patients with hematologic malignancy and $47.1 \%$ in the patients with solid tumor.

The increase in ESBL producing strains has caused more use of carbapenems and this has ended up with increased resistance (18). In a study conducted by Trecarichi et al. (15), it was reported that carbapenem resistance could reach up to $38 \%$ in the patients with hematologic malignancy. In this study, carbapenem resistance was found to be $17.9 \%$ in the patients with hematologic malignancy and $5.9 \%$ in the patients with solid tumor. The rate of multidrug-resistant gram-negative bacteria was higher in patients with hematologic malignancy.

In the United States between 2005 and 2017, 55\% reduction in MRSA was observed following the introduction of multifaceted infection control measures in $S$. aureus infections (19). Worldwide, the prevalence of methicillin-resistant $S$. aureus in bloodstream infection is $<1 \%$ in Scandinavia, $40 \%$ in Japan, Israel and Europe (20). In this study, the rate of Grampositive bacteria was lower in both patient groups. The number of methicillin-resistant agents was $44(84.6 \%)$ in the patients with hematologic malignancy and $10(62.5 \%)$ in the patients with solid tumor.

Candidiasis is an important nosocomial infection which is seen both in adults and in children, especially in hospitalized patients $(21,22)$. And common source of infection is the endogenous flora of the patient (23). The risk factors for candidemia may include age, trauma, or burns, as well as CVC, TPN, broad-spectrum antibiotics use, high APACHE scores, acute renal failure, hemodialysis, history of surgery, especially abdominal surgery, gastrointestinal system perforations, and anastomotic leaks (21).

In this study, the above-mentioned risk factors were identified as risk factors for candidemia in both patient groups. Candida growth was found to be very low in both groups. There were five growths in the patients with hematologic malignancy and two in the patients with solid tumor.

The current study had some limitations. As a result of its retrospective design, the duration of $\mathrm{CVC}$, duration of steroid therapy and duration of urinary catheter could not be obtained from the health records.

\section{Conclusion}

As a result, risk factors are higher in the development of bloodstream infection in the patients with hematologic malignancies. Therefore, the patients with hematologic malignancies should be closely monitored for developing blood stream infections. Reducing these risk factors may contribute to reduce the incidence of bloodstream infection. Multidrugresistant bacteria might also be isolated from the blood cultures of the patients with cancer. Therefore, whenever it is necessary, 
should be kept in mind it is recommended to start with broadspectrum antibiotics in bloodstream infections in patients with cancer.

\section{Acknowledgments}

We would like to thank Melih Uzunoğlu for his support in statistical analyses.

\section{Ethics}

Ethics Committee Approval: Ethical approval was obtained from Clinical Research Ethics Committee of University of Health Sciences Turkey Ankara Dr. Abdurrahman Yurtaslan Training and Research Hospital (approval date: 02/20/2019 and decision no: 2019-02/200).

Informed Consent: Retrospective study.

Peer-review: Externally and internally peer-reviewed.

\section{Authorship Contributions}

Concept: D.M., A.M., Design: D.M., M.K.Ç., E.D., Data Collection or Processing: D.M., A.M., N.K., M.K.Ç., E.D., S.Ç., F.A., M.E., Analysis or Interpretation: D.M., N.K., S.Ç., F.A., Literature Search: D.M., Writing: D.M.

Conflict of Interest: No conflict of interest was declared by the authors.

Financial Disclosure: The authors declared that this study received no financial support.

\section{References}

1. Kang Cl, Kim SH, Park WB, et al. Bloodstream infections caused by antibiotic-resistant gram-negative bacilli: risk factors for mortality and impact of inappropriate initial antimicrobial therapy on outcome. Antimicrob Agents Chemother. 2005;49:760-766.

2. Marín M, Gudiol C, Ardanuy C, et al. Bloodstream infections in neutropenic patients with cancer: differences between patients with haematological malignancies and solid tumours. J Infect. 2014;69:417-423.

3. Montassier E, Batard E, Gastinne T, Potel G, de La Cochetière MF. Recent changes in bacteremia in patients with cancer: a systematic review of epidemiology and antibiotic resistance. Eur J Clin Microbiol Infect Dis. 2013;32:841-850.

4. Fridkin SK. The changing face of fungal infections in health care settings. Clin Infect Dis. 2005;41:1455-1460.

5. Safdar A, Armstrong D. Infections in patients with hematologic neoplasms and hematopoietic stem cell transplantation: neutropenia, humoral, and splenic defects. Clin Infect Dis. 2011;53:798-806.

6. Hübel K, Hegener K, Schnell R, et al. Suppressed neutrophil function as a risk factor for severe infection after cytotoxic chemotherapy in patients with acute nonlymphocytic leukemia. Ann Hematol. 1999;78:73-77.

7. Obeng-Nkrumah N, Labi AK, Acquah ME, Donkor ES. Bloodstream infections in patients with malignancies: implications for antibiotic treatment in a Ghanaian tertiary setting. BMC Res Notes. 2015;8:742.

8. Graff LR, Franklin KK, Witt L, et al. Antimicrobial therapy of gram-negative bacteremia at two university-affiliated medical centers. Am J Med. 2002;112:204-211.

9. Vidal F, Mensa J, Almela M, et al. Bacteraemia in adults due to glucose non-fermentative Gram-negative bacilli other than P. aeruginosa. QJM. 2003;96:227-234.

10. Shmuely H, Pitlik S, Yahav J, Samra Z, Leibovici L. Sevenyear study of bacteremia in hospitalized patients on chronic hemodialysis in a single tertiary hospital. Ren Fail. 2003;25:579-588.

11. Marschall J, Zhang L, Foxman B, Warren DK, Henderson JP; CDC Prevention Epicenters Program. Both host and pathogen factors predispose to Escherichia coli urinarysource bacteremia in hospitalized patients. Clin Infect Dis. 2012;54:1692-1698.

12. Safdar A, Armstrong D. Infectious morbidity in critically ill patients with cancer. Crit Care Clin. 2001;17:531-570.

13. Velasco E, Soares M, Byington $R$, et al. Prospective evaluation of the epidemiology, microbiology, and outcome of bloodstream infections in adult surgical cancer patients. Eur J Clin Microbiol Infect Dis. 2004;23:596-602.

14. Ruhnke M, Arnold R, Gastmeier P. Infection control issues in patients with haematological malignancies in the era of multidrug-resistant bacteria. Lancet Oncol. 2014;15:606619.

15. Trecarichi EM, Pagano L, Candoni A, et al. Current epidemiology and antimicrobial resistance data for bacterial bloodstream infections in patients with hematologic malignancies: an Italian multicentre prospective survey. Clin Microbiol Infect. 2015;21:337-343.

16. Islas-Muñoz B, Volkow-Fernández $P$, Ibanes-Gutiérrez C, Villamar-Ramírez A, Vilar-Compte D, Cornejo-Juárez P. Bloodstream infections in cancer patients. Risk factors associated with mortality. Int J Infect Dis. 2018;71:59-64.

17. Miedema KG, Winter RH, Ammann RA, et al. Bacteria causing bacteremia in pediatric cancer patients presenting with febrile neutropenia-species distribution and susceptibility patterns. Support Care Cancer. 2013;21:2417-2426.

18. Peralta G, Lamelo M, Álvarez-García P, et al. Impact of empirical treatment in extended-spectrum beta-lactamaseproducing Escherichia coli and Klebsiella spp. bacteremia. A multicentric cohort study. BMC Infect Dis. 2012;12:245. 
19. Jones M, Jernigan JA, Evans ME, Roselle GA, Hatfield KM, Samore MH. Vital Signs: Trends in Staphylococcus aureus Infections in Veterans Affairs Medical Centers United States, 2005-2017. MMWR Morb Mortal Wkly Rep. 2019;68:220-224.

20. Sader HS, Streit JM, Fritsche TR, Jones RN. Antimicrobial susceptibility of gram-positive bacteria isolated from European medical centres: results of the Daptomycin Surveillance Programme (2002-2004). Clin Microbiol Infect. 2006;12:844-852.
21. Kullberg BJ, Arendrup MC. Invasive Candidiasis. N Engl J Med. 2015;373:1445-1456.

22. Kollef M, Micek S, Hampton N, Doherty JA, Kumar A. Septic shock attributed to Candida infection: importance of empiric therapy and source control. Clin Infect Dis. 2012;54:1739-1746.

23. Pfaller MA, Diekema DJ. Epidemiology of invasive candidiasis: a persistent public health problem. Clin Microbiol Rev. 2007;20:133-163. 